\title{
A Discussion with Fields Medalist Artur Avila
}

Michael Th. Rassias (University of Zürich, Switzerland)

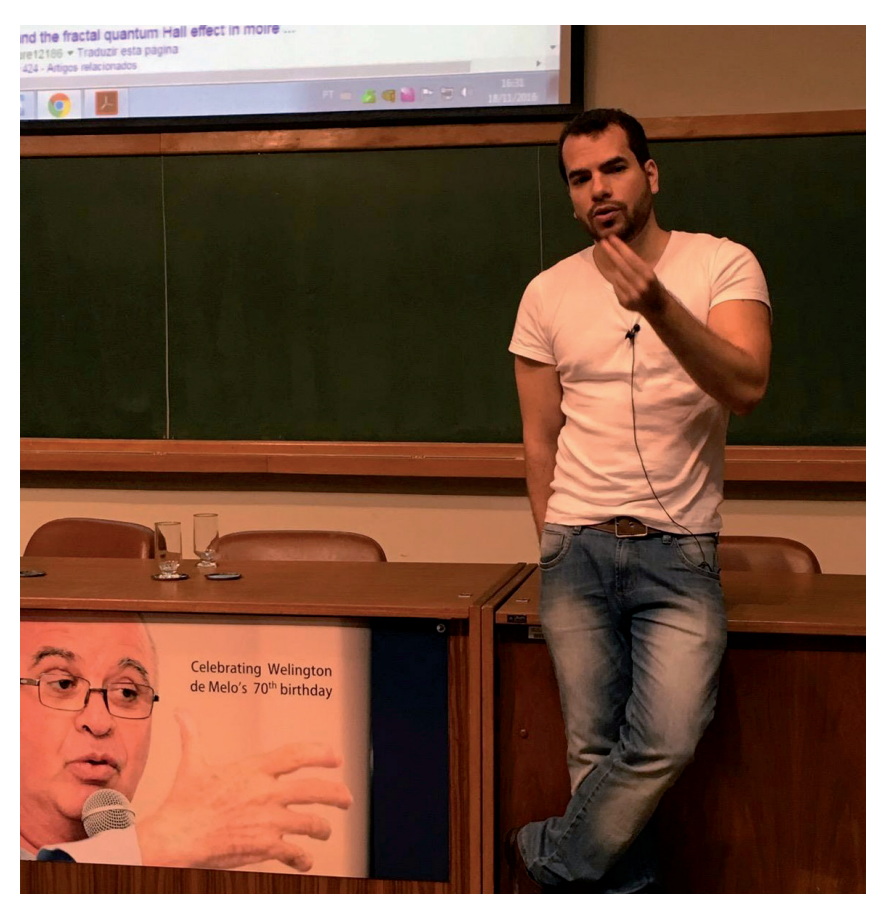

Artur Avila, born in 1979 in Rio de Janeiro, Brazil, is a world-renowned mathematician with outstanding contributions in a broad spectrum of topics, with his main work focusing on dynamical systems. His problem-solving mastery, technical power and ingenuity have led him to the proof of famous and long-standing open problems like the "Ten Martini Problem" and the "Zorich-Kontsevich conjecture", to name just a few. He was awarded the Fields Medal in 2014, being the first Latin American to win this award. He recently joined the University of Zürich, Switzerland, after spending years as a researcher in the CNRS (France), and remains associated with IMPA (Brazil) where he got his PhD in 2001.

Honoured to be working under the same academic roof in Zürich, I had the great privilege of meeting Artur a few months ago and spending some time with him discussing various topics. With this opportunity the following discussion arose, in which he talks about the path of his mathematical trajectory, as well as his interesting views on certain issues.

\section{I know you participated in an IMO and won a gold medal. From which age did you get interested in math- ematics?}

My particular interest for mathematics started pretty early on, from the age of 4 or 5. I tried to learn beyond what was taught at school and I would ask for books in order to read by myself. Since my parents were not academics and would not know which books would be good to buy for me, there was some kind of a random process of buying several books, some good and some not, which were my original source of self-study. I was also very interested early on in the concept of science, like Physics, chemistry, etc., even in the primitive way I could understand it as a child. But at the time I certainly had no understanding that being a mathematician could be a career choice. It somehow seemed a bit more detached from the other sciences. By the nature of the field, what you are usually initially presented with is very old stuff. You don't see modern discoveries, whereas in physics for example, as a kid you hear about relativity and things that you can associate with recent discoveries and thus feel that the field is active. On the other hand, you might often get the impression that maths has been done by people in the past and there is nothing new to do. So the concept of becoming a mathematician did not initially exist.

Do you think that this might be related to how mathematics is taught in Brazil or is it more general?

I think that this is a more general difficulty that mathematics has with respect to other sciences. This happens because in mathematics we always build upon foundations. For example, what Euclid did is still valid and that is why we study it until today, whereas we don't try to learn physics, say, according to Aristotle, as that was completely wrong. In physics we are being taught more modern things. Maybe we start with Galileo, but we don't go back to Aristotle's time, while in mathematics we do start with Euclid. It is the nature of the subject to a certain extent. We go further back. So I don't think that it is mainly a matter of geography.

\section{So how did you get more actively involved with math- ematics?}

At the age of 13, I heard about the existence of a national mathematical olympiad in Brazil without knowing exactly what that was. At the time I was reading calculus books and related things, but I was not familiar with this type of problem-solving. I got in touch with it and I thought that it was interesting. My first encounters were difficult but then I got really involved for a few years. That was my new impression of what mathematics could be. In Brazil olympiads are organised by IMPA to a large extent. Being from Rio De Janeiro, my fist contact with IMPA occured when I went there to collect a medal, as IMPA is also in Rio. So that is how I found out about its existence and the fact that research was done there. Also, IMPA has a very flexible system which even allows for high school students to study there and obtain their master's. So it can happen that a student starts a master's at the age of 16 and 


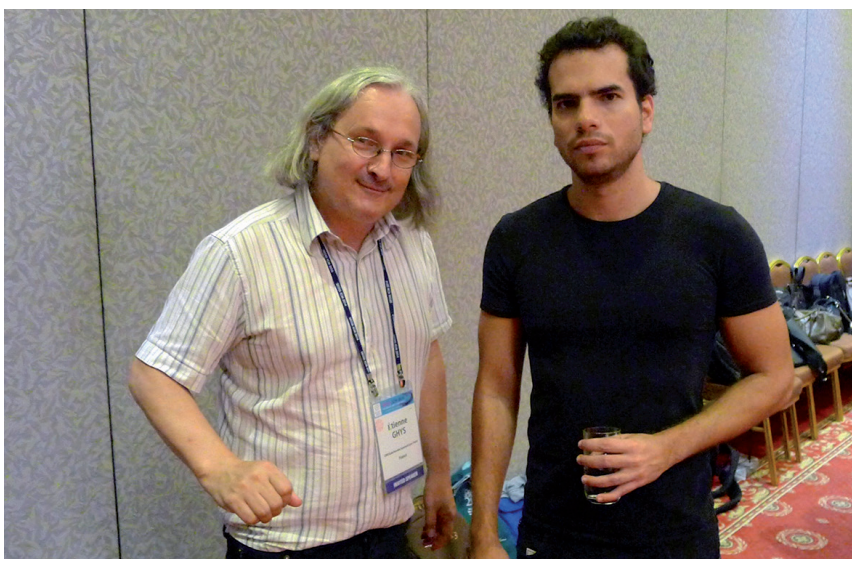

Etienne Ghys (left) with Artur Avila (right) at the 2014 ICM in Seoul. (Photo by Joachim Heinze, Archives of the Mathematisches Forschungsinstitut Oberwolfach)

finishes it at 18 and then obtains his $\mathrm{PhD}$ at the age of 21. There are examples like that.

You are one such exceptional example. You started your PhD studies at the very young age of 19 at IMPA, correct? So yes, when I found out about the existence of IMPA I thought it would be nice to get involved. In my mind, I imagined that it could probably help me with my olympiads training. Once I got a gold medal at the IMO I got an invitation from IMPA to take part in a pre-master's programme: a sort of scientific initiation program. It was a special course called "Introduction to Topology". I got a fellowship, I did well and then started the master's programme while in the last year of high school. Subsequently I commenced my PhD there as well.

Do you believe that the strategic way of thinking you had cultivated as an olympiad caliber problem-solver helped you later when approaching problems on a research level?

The olympiads gave me confidence to start with. So when I started the master's programme I felt that it was something I could really do and I worked intensively for it. But in research you learn how to think in a different manner. Of course tricks and little techniques inherited by the olympiads may be helpful not to waste time occasionally as you may recognise easily that something might fail to work, but in research you have to think about a problem whose solution is unknown. In competitions, the problems are designed to be solved. There is of course creativity involved, but the problems are such that they can be solved by the use of a specific toolbox. In research you deal with uncertainty. You also have to be able to recognise whether you should continue on a certain path or maybe start researching a little bit to the side, and know when to come back to the original problem again.

\section{What is your advice to young talented students in Brazil and elsewhere?}

I feel that the students have to take so many courses at the university that they have no time to engage at a deeper level with any of them. With so many required classes per week, they can only realistically strive for su- perficial understanding. It would be better to focus more on something more specific and understand it well. But of course, the question is where to focus. I advise students to try to identify, as soon as they can, what attracts them most and then put more energy into that, since you have to concentrate on something in order to understand it and you cannot achieve that by sharing time equally between several subjects. Unfortunately, the system is not designed to allow you to do this. Of course, it is easier to observe where things go wrong than to suggest how they could be done better. I would suggest to students to identify a professor and try to get into contact with him/ her in order to get guidance.

You recently joined the University of Zürich (UZH), while prior to that you were in Paris. How do you find the new environment here?

The environment and the mathematical centre in Zürich is very active. There is a very good synergy between the UZH and ETH. An increasing number of good researchers stay here as well as just pass by, and thus the seminars are very active. Even though Paris, for example, has many more people, one can say that to some extent after a certain size of community you cannot really absorb everything that is going on. It is impossible to attend all of the seminars, meet with all of the people, etc. The nice thing about Zurich is that the size is such that you can absorb what happens and there is a good number of things happening. At the UZH we increased the dynamics group as well. Apart from me, two other researchers from Bristol have joined. We also established an "Ergodic theory and dynamical systems" seminar that is already becoming very active. We hope that it will be one of the central seminars in dynamical systems in Europe.

Was there a specific paper, book, lecture, or even a theorem you came across that had a lasting impact on you to the extent that it made you chose to become a mathematician?

In my case it was a long experience and a very gradual process. There were many things that grabbed my interest and several theorems that I liked while growing up. Additionally, my mental understanding of what mathematics is progressed over the years and I realise now that I was fortunate, since there were several stages in my development where the process might have not worked out so well. In general, a student might like olympiads and really enjoy problem-solving but could not be so attracted by the theory learning that happens at, say, in master's program. Or they may like learning all those wonderful theorems, but might not find it pleasurable to try and prove new theorems. Eventually, what leads you to becoming a researcher is a path that comes from many different directions. But there are several little things in the process that might not work out for someone and lead them elsewhere.

So in my case there was no particular theorem or open problem that lead me to mathematics, such as Fermat's last theorem, Poincaré's conjecture, etc. It was a long and gradual process. 
What is the first thing that comes to mind when you are thinking of the word "mathematics"?

Generally, my way of thinking is that of an analyst. So, when I think of "mathematics" the first things that come to mind are "analysis" and "inequalities". Inequalities are really central to my way of thinking.

Is there a mathematician who influenced you the most? Either through your mutual collaboration or interaction or even by studying his work?

There were several people who were important and influential. In research you have your own style, but it is very good to be exposed to several other views and possibilities. There is also a lot of advice you can get. You can of course choose to adopt some advice and some not. My PhD advisor Welington de Melo was influential to me. I was also influenced by Mikhail Lyubich, with whom I worked a lot. Later there was also Jean-Christophe Yoccoz, whose style was very different. These mathematicians had their own particular styles and I was exposed to that as I interacted with them directly. Those were close to me, but of course indirectly I was influenced by more distant people. For example, when I was shifting a bit between fields, I found Jean Bourgain's work to be an inspiration. His style and abilities sparked some admiration for the corresponding fields. His style of doing analysis is something I like very much and I have the highest respect for him.

You started your PhD studies at the very young age of 19 at IMPA. Your doctoral advisor there was Welington de Melo who was working on dynamical systems and particularly on one-dimensional dynamics, which is a very demanding field. Did he allure you somehow towards that dynamical systems or had you already chosen your path?

When I initially went to IMPA I had a very weak background and I was just finding out about the possibility of doing research. I wanted to learn the basics at the time and I was concentrated on trying to do well at my Master's programme. During that period I attended a PhD course in differential topology by de Melo. I was not very keen on attending that course at first, as de Melo had the reputation of being very tough with students and it was considered difficult to get a good mark in his courses. But it went well and he liked me. After the completion of the course he approached me and he proposed that we discuss some topics in complex analysis, quasiconformal mappings, etc. Through that process he naturally became my advisor. At IMPA, dynamics was a top field and they were strong at it. De Melo was a bit isolated, though. He was working on one-dimensional dynamics: a field that had already become very important but was and remains very hard. So one had to put all ones energy into this specific field and it made it hard to interact with others working on different things. The techniques and main problems are very difficult. An example of the complications involved is the following: we often have to work with fractal sets with very complicated combinatorics, and just to get the correct language to describe their structure is very hard. In analysis in some cases you might

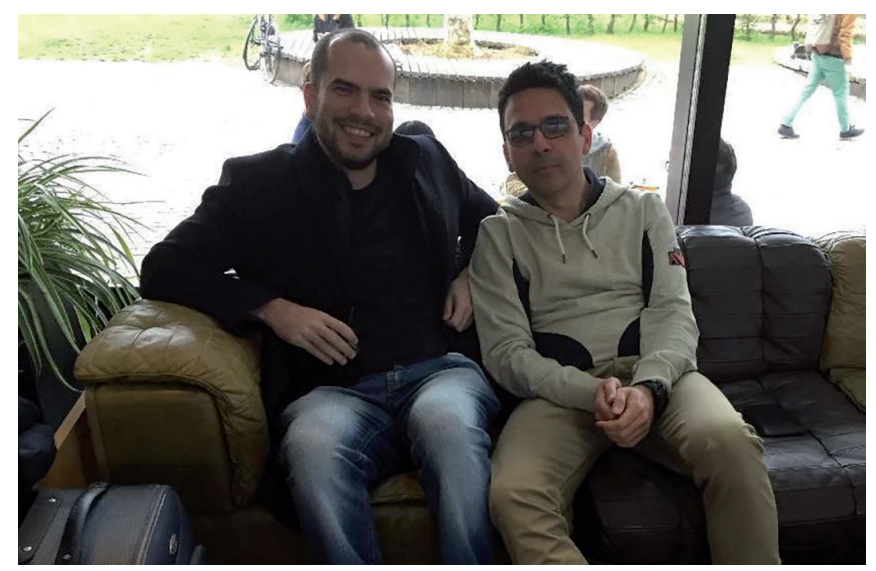

Artur Avila (left) with Ashkan Nikeghbali (right) at the University of Zürich.

just take a dyadic division, break everything into squares and so on. But here you will need to do this in fractal sets, and just the description of the pieces is complicated. You have to describe the set and the topology is very intricate. For this reason, until today, whenever I have to supervise $\mathrm{PhD}$ students I tell them that if you do one-dimensional dynamics you will have to put all your effort and energy into that particular field, which is very specialised. Otherwise, we can cover almost everything else in dynamics. So I often propose to them to follow the second option, as it might not be a very good idea to get too specialised so early on. Of course, when I started I did not know any of this, and I just said OK and worked on one-dimensional dynamics. It turned out to be very interesting and very hard as well. Later I learned more things and expanded in other directions too. I still work on one-dimensional dynamics, but now most of my work is on other things.

One may state that mathematics has witnessed great expansion during the last, say, one hundred years, with many different areas emerging and various methods discovered, bridging seemingly different fields. How do you see the future of mathematics in that respect? Do you think that interdisciplinarity might be the theme of the future for example?

Things tend to become more and more technical and thus people get further apart as they don't speak the same language. It has become clear that for an analyst it is more difficult to understand what they are now doing in algebra. My view on this is that people should just do what they want. If someone wants to think about prime numbers he should do that, if he wants to think about fractals then that is fine as well. Some researchers will make progress working on isolated topics and others might want to bridge together areas and examine the connections between discoveries. Not everyone has to do that. Some people might just want to play on their own. You cannot anticipate what is going to work out. It is also not necessary to decide which fields of mathematics have to be stimulated, since we don't know whether the next discovery in geometry will depend on a discovery in algebra and so on.

This is also my point of view regarding focusing on pure or applied mathematics. What might be crucial in 
the solution of some very specific problem (with industrial applications or whatever) often happens to be just some beautiful object that someone studied for its own sake. The motivation of the latter (often similar to the motivation a child has for playing a game) is irrelevant to the fact that what has been discovered turns out to be useful to the former. And of course it works both ways: much of my work deals with theories that first arose in connection to physics, but for me what matters is that these theories are rich mathematically, and I study them from this perspective. So "pure" mathematicians have to recognise that many of the nice objects they have at their disposal with rich mathematical theorems that one works on just because they are beautiful, without caring about the motivation or the application, might have not been discovered if it was not for some physical model that someone was examining because they cared about the physics of it. Thus, by admitting this and letting people work on what they want, the whole community benefits from the eventual (unpredictable) interactions.

It has happened many times in the past that great mathematicians moved from area to area, making contributions to various different mathematical domains. Stephen Smale is one such example, part of whose work is on dynamical systems like yours, but who has also worked in topology and mathematical economics with the latter being fairly distant from the other two fields. Do you see yourself exploring completely new research terrains in the future?

With Smale there was a natural evolution from topology to dynamical systems, since part of his work towards Poincare's conjecture was already leading him to understand certain types of dynamical systems. Sometimes of course there is an explicit desire for one to do something different. So I believe that Smale actually forced himself to turn to another direction. In my case, things happened to evolve in directions that I had not anticipated. It has happened that I have turned my attention to something which I found attractive and later it turned out to be related to things I had been doing. The connection proved to be deeper than I would initially have expected. I assume that this will tend to continue to happen. Personally, I do not put in a great effort to work on different areas, except if I find something attractive and there is a natural progression that leads me there.

On a humorous note, if - like in the case of Ramanujan - there were a supreme being that could hand you solutions to important problems in your sleep, and say that you could have only one such dream, which problem would you like to see the solution for?

$\mathrm{Mm} .$. The first thing that comes to mind is the local connectivity of the Mandelbrot set. I don't know whether I would change my mind if I put more thought to into it, but this is the first problem that I thought of when you asked me this question.

I don't actively work on it. I could possibly work on it in the future if the corresponding techniques have evolved, but it is certainly a beautiful problem that is captivating.

Many scientists from a broad spectrum of areas have expressed various views and opinions about the possible future consequences of the advancement of artificial intelligence (AI) in connection to the so-called "AIcontrol problem". Being a young scientist, during whose life it is very likely that great advances in AI might be achieved, affecting our lives, do you have any views on this?

Being a dynamicist, my training has taught me to a large extent that there are a lot of limitations when we make predictions. To my understanding, when it comes to AI peoples' hopes might be too high at the moment, as one tends to get too optimistic following a few unexpected successes. It is of course correct to continue the effort, as probably there is more success to come, but it is reasonable to imagine that obstacles will be identified so that things will not advance as fast as it was hoped. Even though it has happened many times in science that several things that we didn't think could be done in a specific timeframe were actually achieved and we were very surprised by the outcome, we should not get too carried away. So, I am not particularly scared about the consequences of the advancement of AI, since expecting big advancements in $\mathrm{AI}$ is already large assumption. Expecting that AI will become something applicable to almost all situations involves too much wishful thinking, even in the long run.

When we learn more about a corresponding domain we are also able to better understand its limitations. The point is to try to discover directions where the techniques allow you to go. It is very important and at the same time difficult to know what you cannot achieve. A lot of good quality research is conducted to see exactly what is impossible to be done, in order to know what the barriers of the techniques are. Barriers that will not be overcome, since they are theoretical ones. That is, it is important to know that it is not a matter of putting more effort towards a goal if that goal is really intractable. This is what happened in mathematics when we learned that there are unsolvable problems and all kind of limitations to formalism. Our understanding changed completely from Hilbert to Gödel, could this have been predicted? In technology, while there was very fast progress with the clock speed of chips, we could anticipate that this needed to stop at some level as there were known physical limitations. Maybe, with respect to AI, there are still unknown theoretical limitations hiding in information theory or even dynamical systems.

Michael Th. Rassias is on the Editorial Board of the EMS Newsletter. His photo and $C V$ can be found in previous Newsletter issues. 